Rabaska

Revue d'ethnologie de l'Amérique française

\title{
Centre de folklore acadien et créole (Université de Louisiane à Lafayette)
}

\section{Barry-Jean Ancelet}

Volume 9, 2011

URI : https://id.erudit.org/iderudit/1005956ar

DOI : https://doi.org/10.7202/1005956ar

Aller au sommaire du numéro

\section{Éditeur(s)}

Société québécoise d'ethnologie

ISSN

1703-7433 (imprimé)

1916-7350 (numérique)

Découvrir la revue

Citer ce document

Ancelet, B.-J. (2011). Centre de folklore acadien et créole (Université de

Louisiane à Lafayette). Rabaska, 9, 389-391. https://doi.org/10.7202/1005956ar d'utilisation que vous pouvez consulter en ligne.

https://apropos.erudit.org/fr/usagers/politique-dutilisation/ 
Centre de folklore acadien et créole

Center for Acadian and Creole Folklore

University of Louisiana at Lafayette

Lafayette, LA 70504

Téléphone : (337) 482-6811

Télécopieur : (337) 482-5446

Courriel : ancelet@louisiana.edu

Toile : http://languages.louisiana.edu/French/centeracadian.html

\section{Mandat et collections}

Le Centre d'études louisianaises, qui parraine le CFAC, vient d'avoir un nouveau directeur, Michael Martin, professeur d'histoire, qui remplace Carl Brasseaux, qui a pris sa retraite pendant l'année. Le CFAC a aussi engagé Chris Segura, membre du groupe Feufollet, en tant qu' archiviste-technicien. Chris Stafford, qui joue aussi dans Feufollet, est assistant-technicien. Marc Dewitt vient d'être nommé professeur d'ethnomusicologie, occupant la nouvelle chaire Tommy-Comeaux, nommée en mémoire de ce musicien traditionnel. Le Centre (CFAC) continue dans son esprit de « recyclage culturel», mettant ses collections historiques et contemporaines à la disposition du public (chercheurs, musiciens, cinéastes, enseignants, etc.), et organisant aussi des festivals et des spectacles spéciaux, des émissions de télévision et de radio. Il dispense des cours et des ateliers au sein des programmes universitaires, surtout en Études francophones, en Histoire, en Musique et en Anthropologie culturelle. Le CFAC produit des documents - livres et articles, disques et vidéos - qui diffusent ces créations et ces réinterprétations auprès de la communauté et des étudiants. Il explore des façons de vitaliser la francophonie en Louisiane. Il a régulièrement recours à des chanteurs et à des conteurs, comme à des conférenciers universitaires, qui contribuent aux programmes d'enseignement. Il participe toujours à la production des Festivals acadiens et créoles, soit la deuxième fin de semaine d'octobre chaque année.

Les Archives de folklore acadien et créole poursuivent leurs efforts pour stabiliser et préserver les enregistrements des collections qu'elles conservent. On en a copié une partie importante en format numérique qui peut être consultée par ordinateur, y compris récemment les enregistrements des Festivals acadiens et créoles. On continue à numériser des photos de plusieurs collections, y compris celle d'Ancelet. On commence à intégrer des collections d'étudiants, non seulement de l'Université de Louisiane, mais aussi d'autres institutions de l'État. Nous venons aussi de recevoir une collection manuscrite de Carol Moore, représentant des travaux d'étudiants sénégalais sur la tradition orale dans le pays durant les années 1960. Erin Stickney, étudiante de $3^{\mathrm{e}}$ cycle en Études francophones, y œuvre à titre d'assistante de recherche cette année pour identifier et transcrire les ressources d'expression française.

Projets en cours

Nous continuons à travailler sur la tradition orale - contes et chansons - en Louisiane, 
établissant des corpus pour éventuellement examiner des éléments récurrents dans le bagage symbolique. Barry Ancelet, Marcia Gaudet et Carl Lindahl (de l'Université de Houston) achèvent Storms Stories, une anthologie de témoignages et d'histoires concernant les ouragans récents (surtout Katrina et Rita) ; Ancelet, Gaudet et Ray Brassieur continuent de documenter l'évolution de l'architecture vernaculaire en réponse à ces mêmes événements. Ancelet et May Waggoner achèvent une anthologie de la littérature d'expression française en Louisiane, des débuts de la colonie jusqu'au début du $\mathrm{Xx}^{\mathrm{e}}$ siècle. Gaudet est devenue directrice du nouveau centre Ernest-Gaines, nommé en l'honneur de l'écrivain créole louisianais. Brassieur travaille toujours sur l'artisanat, surtout les bateaux, sur les relations entre les groupes ethniques en Louisiane, sur les plantes médicinales en Louisiane ; il vient de lancer un projet pour étudier la communauté créole de La Terre Promise le long du bayou Tèche. John Laudun continue ses recherches sur la culture matérielle en Louisiane, sur les bateaux. Les membres du Centre préparent une série de disques à partir de nos collections ; signalons Walter Mouton : Live at Festivals Acadiens; Zachary Richard : Live at Festivals Acadiens, 1981 ; et Festivals Acadiens : Live, 2002.

Plusieurs membres continuent leurs recherches sur le Mardi Gras en Louisiane, et participent à des interprétations de plusieurs genres (expositions, publications, festivals, etc.). Ancelet a présenté une conférence sur le Mardi Gras en Louisiane au Center for Research in Festive Culture, Newberry Library, à Chicago, ainsi qu'une série de conférences sur les traditions louisianaises à l'Université de Moncton, à l'Université de Poitiers et à l'Université Laval. Nos recherches sur la poétique de la chanson cadienne et créole se poursuivent. Ces informations, nos transcriptions, nos traductions et nos annotations sont souvent utilisées par des musiciens de la région pour leurs projets de disques. Les membres du CFAC communiquent régulièrement les résultats de leurs recherches non seulement aux colloques des sociétés savantes (en histoire, linguistique, littérature, ethnologie, folklore, etc.), mais aussi dans des présentations publiques dans la région. Jennifer Ritter continue à organiser la série In Your Own Backyard, où les membres de notre équipe peuvent communiquer leurs recherches au public louisianais. ( $c f$. le site ccet.louisiana.edu). Cette année, les Festivals acadiens et créoles intègrent le Grand Réveil acadien, une semaine de rencontres et de performances mettant en évidence les liens entre la Louisiane et les provinces maritimes du Canada.

Ancelet a fait partie de la tournée « Contes de la Louisiane : Zistwar : La nuit du conte », organisée par la délégation générale des Alliances françaises, le Consulat de France et le Centre des francophones d'Amérique, qui a joué à San Francisco, Atlanta, New-York, Washington, Chicago, Montréal et Québec ; ainsi qu'aux Fêtes de la Nouvelle-France à Québec.

\section{Projets média}

Ritter organise régulièrement aussi une série de films ethnographiques, dont la projection est suivie d'une table ronde. Ancelet a présenté Tootie 's Last Suit (2008) et Tee Mamou (2011) au Cinema on the Bayou Film Festival, et il a servi de consultant pour J'écris l'image (de Daniel Rocque), un documentaire sur le statut du français en Louisiane, ainsi que pour Still Singing the Blues, un documentaire radio (de Richard 
Ziglar et Barry Yeoman; Filmmakers Collaborative/Louisiana Endowment for the Humanities).

\section{Honneur}

Le Dictionary of Louisiana French a été nommé livre de l'année 2010 par le Louisiana Endowment for the Humanities.

Activités des étudiants

- Thèse achevée : Josh Caffery, « Ride les Blues : The Lomaxes in Coastal Louisiana, 1934 ». - Thèses en préparation: Madeline Padgett (sur la pédagogie et la technologie), Valérie Broussard (sur la littérature et la culture cadiennes en Louisiane), Jean-Marie Ntipouna (sur l'influence de l'Ouest sur l'éducation au Cameroun), Fatoumata Modi (sur la transition de l'oral à l'écrit dans la tradition de l'épopée au Niger). - Mémoire en préparation : Ferran Bertrand (sur la tradition orale dans une famille en Louisiane).

BARRY-JEAN ANCELET 